\title{
Generating Logistic Characteristic Curves using Discrete Event Simulation and Response Surface Models
}

\author{
Sonja Kuhnt ${ }^{1}$, Dominik Kirchhoff ${ }^{1}$, Sigrid Wenzel ${ }^{2 *}$, Jana Stolipin ${ }^{2}$ \\ ${ }^{1}$ Department of Computer Science, FH Dortmund University of Applied Sciences and Arts, Emil-Figge-Str. 42, \\ 44227 Dortmund, Germany; \\ ${ }^{2}$ Department of Production Organization and Factory Planning, University of Kassel, Kurt-Wolters-Str. 3, \\ 34125 Kassel, Germany; s.wenzel@uni-kassel.de
}

SNE 30(3), 2020, 95-104, DOI: 10.11128/sne.30.tn.10522 Received: August 4, 2020; Revised: August 15, 2020; Accepted: August 20, 2020

SNE - Simulation Notes Europe, ARGESIM Publisher Vienna, ISSN Print 2305-9974, Online 2306-0271, www.sne-journal.org

Abstract. Logistic Characteristic Curves (LCCS) or Logistic Operating Curves (LOCS) describe relationships between various Key Performance Indicators (KPIs) of production and logistics systems. These relationships can be qualitatively or quantitatively visualized by charts to illustrate the performance of these systems. Discrete Event Simulation (DES) allows a detailed investigation of the dynamic behavior of production and logistics systems under consideration of uncertainties and thus contributes to their planning reliability. Using simulation models and the data generated by the experiments, KPIs of the modeled systems are measured. Of course, different production and logistics systems also have several target systems whereby the individual target variables interact with each other and can, therefore, conflict. In this paper, a methodology is presented that combines DES and a statistical technique for empirical model building, namely the response surface model, to predict the behavior of production and logistics systems by using LOCS and thereby decrease the effort for experimentation by reducing the number of simulation runs.

\section{Introduction}

Logistic Characteristic Curves (LCCs) graphically show the qualitative and quantitative interrelationships between logistic key performance indicators (KPIs) in an in-house production and logistics systems.
Often, different logistic KPIs are in a field of conflict between logistic performance and logistic costs, and it is impossible to achieve a system optimum for all of them at the same time. Generally, the goals given for a planning task must be positioned within the logistic KPIs. LCCs, also known as Logistic Operating Curves (LOCs), provide a tool for supporting this process $[1,2]$. In difference to performance indicators, which can also be measured in a running production process, complete operating curves can only be determined by mathematical or simulation models, since experiments on the real system are not possible for economic reasons.

In the field of production and logistics system planning, Discrete Event Simulation (DES) is a recognized problem-solving method [3] and, therefore, also an important Digital Factory method [4]. DES supports the detailed examination of production and logistics systems with discrete unit loads by analyzing the dynamic and random behavior to uncover possible planning errors and inaccuracies in behavior. Simulation offers the possibility to examine a system without developing or disturbing real systems, so that process alternatives can be checked, system structures can be varied, and different controls and parameter settings of systems can be tested. The better the data situation of a simulation study is (i.e., the output data of the experiments), the more precise are the statements about the system that are to be made based on the designed experiments. Both the design of suitable experiments and the analysis of the data generated by the simulation models are challenges in a simulation study.

In this paper, we propose to derive LCCs for a simulation model of a manufacturing logistics system by statistical metamodels. 
Thereby LCCs are able to be inferred for complex systems. It is also possible to show the effect of several system parameter configurations at the same time. The general idea behind dealing with expensive function evaluations or simulation experiments by using surrogate- or metamodels is discussed in Section 1, which also deals with the concept of LCCs and DES. In Section 2, we introduce the general approach to derive LCCs from a limited number of well-selected simulation runs by building metamodels for selected logistic KPIs. The approach is exemplified in Section 3 by an application to a manufacturing logistics system with two workstations, as described in the DOSIMIS-3 tutorial [5]. We conclude with a summary and outlook in Section 4.

\section{Background}

\subsection{Logistic characteristic curves}

Using LCCs, the interrelationships between logistic KPIs in in-house production and logistics systems are shown in the form of graphs. The core processes of these systems comprise procurement, production, and distribution $[1,2]$. The LOC theory [6] is built on a deductive-experiment effects model. This model is used as a starting point for the development of approximation equations for the mathematical description of a real logistics system using LOCs. These curves reduce the complexity and, therefore, the costs of modeling the behavior of logistics systems [8]. With the help of LOCs, it is possible to describe KPIs of systems. The principle of mapping the relationships between the effects of the KPIs of production and logistics systems using the LOC theory can be transferred to the production areas for each networked system. LOCs can be used to control and improve the reliability of logistical system performance [1]. LOCs realize effectivity support in the planning and control of operations by giving qualitative and quantitative descriptions of, for example, the KPI Work-In-Process (WIP) and its effects on the output rate and the throughput time. The objectives can be weighted, and then the parameters can be adjusted. An enterprise can adapt its internal requirements in conformity with the market. LOCs make it possible to understand and mathematically describe the interrelationships between the logistic goals such as output rate, lead-time, and WIP [8]. They are an excellent basis for supporting and monitoring the process safety and capability and can be used to evaluate the process during production control. For instance, under the existing structural condition, they can demonstrate that throughput times and WIP value can be reached at one workstation without having to calculate the material flow disruptions or the associated power losses. For a successful implementation of LOCs in production planning and control, goal-oriented system parameters are derived and adapted $[7,8]$. The LOC theory is closely related to the simulation of production and logistics systems. The evaluation of the system performance is a central issue in production planning and control, as well as in simulation and optimization of production and logistics processes. Often, specific parameters are used, which only allow an evaluation in the selected operating point. Unlike the performance parameters, which can also be measured in a running production process, complete operating curves can only be determined through simulation models, since experiments in the real system are not possible for economic reasons. Real logistics systems can be compared with each other using the standardized characteristic curves. In most cases, however, the reference values for this standardization can only be determined using simulation methods [9].

\subsection{Discrete event simulation}

The use of the DES in production and logistics is presented in detail in the series of standards VDI 3633 [10], as well as in [11], [12], and others. DES differs from continuous simulation methods by mapping the progress of time via atomic events that trigger state changes. Each event has a timestamp and is added to an event queue, the simulation time is set according to the timestamp (from the first event in the queue), and then the event is processed [3]. The scope of DES applications ranges from the consideration of in-house manufacturing logistics to procurement and distribution logistics. The evaluation criteria in a simulation study are determined according to the objectives and the object of investigation. Among other things, buffer dimensions and performance data of the machines are evaluated and processing times, transport speeds, or batch sizes are varied in order to determine throughput times and capacity utilization or to reduce transport, or storage times. A simulation-supported examination can prove, for example, whether the planned production quantity can be handled, transported, and produced based on the production concept developed. Within the framework of a simulation study, a model is built using a suitable software tool. 
The simulation models are used in the operating phase, as well as in the planning phase of production and logistics systems. By means of a systematic variation of individual parameters of a simulation model and corresponding experiments, planning concepts can be analyzed according to the defined objectives of a simulation study. To be able to make more precise statements about the systems under investigation, it is necessary to examine a large volume of simulation data. When investigating complex production and logistics systems with comprehensive questions, not only valid models have to be built, but also many complex experiments with a lot of simulation runs must be carried out. The design and implementation of experiments are usually very complicated. However, the generated experimental output data is not the only result of a simulation study. A visualization of the input and output data of the simulation model supports the preparation of the simulation results. The interpretation of the experimental data can be reasonably supported by the methods of statistics and data visualization.

\subsection{Metamodel-based analysis - response surface models}

The word "metamodel" points to the fact that this technique is used to generate a model of a model. A metamodel, or surrogate model, approximates the relationship between the varied input parameters, also called input variables, of a simulation model and so-called response or target variables using a limited number of well-chosen points generated with the simulation model [13]. These points, i.e., settings of the input variables, are determined by a statistical experimental design, which depends on the kind of metamodel and the problem to be solved. Metamodels can be either interpolating or smoothing. The so-called Kriging model is a popular interpolating surrogate model and is most often used for solving expensive black-box optimization problems [13]. It has the ability to not only give point predictions on the target variable, but also to provide the predictive distribution and thereby an uncertainty evaluation over the complete space of input variables. Response surface models, on the other hand, are a class of smoothing models [14]. They can be used for solving optimization problems as well but are especially suitable for the approximation of the unknown relationship between input variables and response variables - the socalled response surface - with minimal quadratic loss.
Given a set of independent input variables $x_{1}, \ldots, x_{p}$, the model has the form $Y=f\left(x_{1}, \ldots, x_{p}\right)+\varepsilon$, where $Y$ denotes the response considered, i.e., in our context one of the logistic KPIs, $f$ is typically a low-order polynomial and $\varepsilon$ a Gaussian error term. Predictions of the expected response achieved by least-square estimates of the unknown parameters of the function $f$, provide LCCs. The accuracy of a fitted response surface model may be evaluated by some additional simulation runs and observing the differences between the outcomes and the predictions given by the model. The finally achieved LCCs not only capture the behavior of the logistic KPIs over the whole domain of different input variables but, moreover, the uncertainty inherent to LCCs can be presented by prediction intervals.

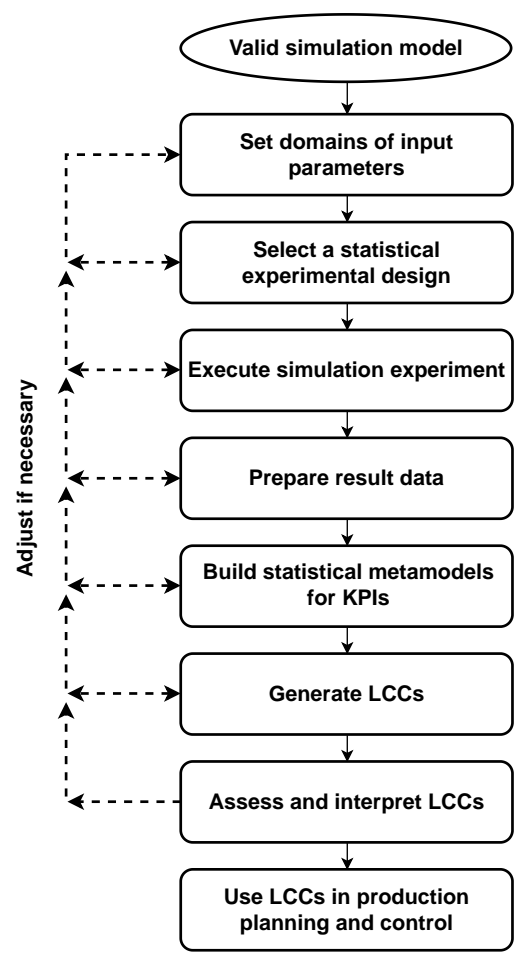

Figure 1: Procedure model for the metamodel-based generation of LCCS 


\section{LCCs from metamodeling of a simulation model}

We now use the metamodel-based analysis of a simulation model to derive LCCs. Our approach is summarized in Figure 1. As a starting point, we assume the existence of a valid simulation model. It must present a reliable image of the real system to be analyzed and return the logistic KPIs of interest as output. We take it that the typical simulation model used in the context of logistics will have some element of uncertainty, e.g., by system loads generated by some statistical distribution. Simulation runs with the same parameter setting but different seeds of the involved random number generator, i.e., replications, then vary in their output. We, therefore, concentrate on response surface models as metamodels in our application in Section 3.

Before we can build a metamodel, we need to discuss which input variables are assumed or known to have an effect on the KPIs and in which range or on which values they can be varied. If we have a list of potential input variables to start with, we might have to conduct a screening analysis first. This can be done by fitting a first-order main effect regression model to the result of a limited number of simulation runs. The primary goal is a rough understanding of the process and to identify the least influential variables by using as few simulation runs as possible. If some of the candidate variables do not display an apparent effect on the measured KPIs, they are set to a fixed value. For the remaining input variables, domains of interest need to be determined, or more specifically, it has to be decided which values or intervals of them are later to be displayed in the LCCs.

Next, a statistical experimental design is set up, which gives the settings of the simulation runs to be done. A more flexible model like a second-order polynomial response surface model with interactions is then to be built from the results of these runs as the basis for the LCCs. Typically, for Kriging models, the methods of choice are so-called Latin Hypercube Designs as they cover the whole domain and thus are called spacefilling designs. Many different experimental designs exist for response surface models. The most popular are two-level factorial and two-level fractional factorial designs for variable screening - i.e., finding out which input variables have an effect on the response and which are negligible.
For the actual response surface fit, often central composite designs, D-optimal designs, or even higherorder factorial designs are the methods of choice [14].

All input variable settings contained in the selected statistical experimental design are then executed. As a result, we have a data set containing measured values for the KPIs for each executed simulation run.

This set of data is subject to an initial analysis to detect possible inconsistencies and get a first impression of structural features. At this point, it might be necessary to return to the beginning of the procedure model (see Figure 1), e.g., if unforeseen effects within the simulation model require an adaption of it.

The next goal is to build a metamodel from the simulation runs, which approximates the true response surface well. A well-chosen metamodel reflects the influences of input parameters and their interdependencies on the KPIs.

LCCs are then created by graphically displaying prediction curves from the response surface (or Kriging) model. To see the effect of different logistic strategies and uses of resources on the logistic KPIs, the predictions are displayed against the varied input variables. Both, response surface models and Kriging models, provide an uncertainty quantification of the prediction, which can be presented as valuable additional information on the plot. While assessing and interpreting the derived LCCs, issues might occur which make a return to previous steps of the procedure model and an adjustment of them necessary, for example, by extending or reducing the range of the input variables. Finally, the procedure model delivers LCCs to be used in planning and control of production and logistics systems.

\section{Demonstration of the procedure model}

\subsection{DOSIMIS-3 simulation model}

For the demonstration of the procedure model described above, the finished basic model of the DOSIMIS-3 tutorial (Figure 2) is used. DOSIMIS-3 is a simulation tool that allows the graphical modeling, visualization, simulation, and analysis of production and logistics systems. The simulation model is described in detail in the DOSIMIS-3 user manual of the SimulationsDienstleistungsZentrum $\mathrm{GmbH}$ [5] and is one part of the corresponding DOSIMIS-3 tutorial. 


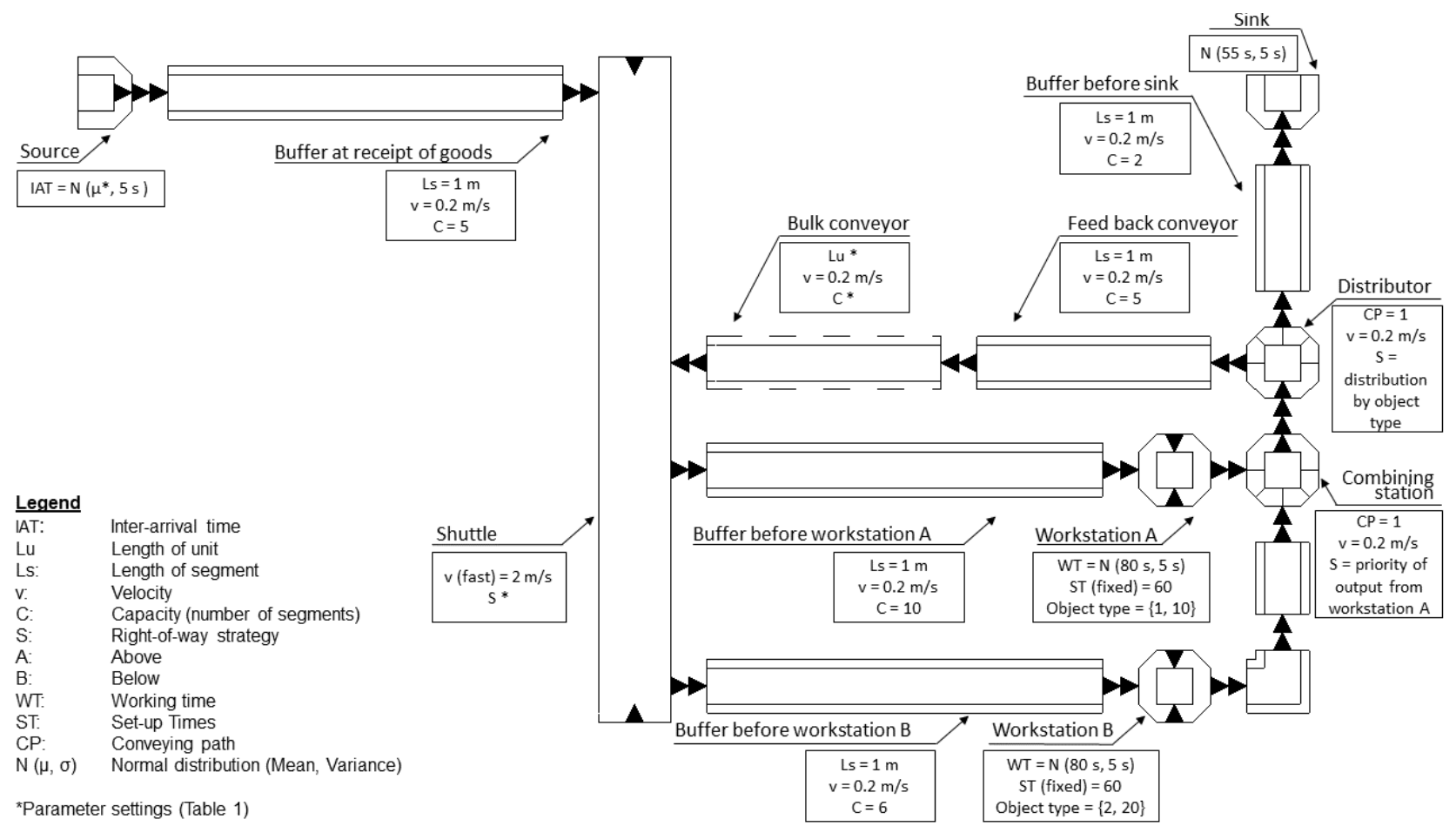

Figure 2: Simulation model in DOSIMIS-3 (for the basic model see [5]).

\begin{tabular}{c|c|c}
\hline Model element & Parameter & Range \\
\hline Source & system load & {$[54,62]$} \\
Shuttle & right-of-way strategy & $\{$ FIFO, Priority to correction route (Prio Corr.) $\}$ \\
Bulk conveyor & batch size & $\{1, \ldots, 8\}$ \\
\hline
\end{tabular}

Table 1: Input variables and selected parameter settings.

The analyzed logistics system is an example of a production process from the electricity industry, which manufactures consumer goods. The borders of this system are the receipt of parts (one source and one queue) and the exit of goods (one queue and one sink). The two product parts (A and B) are randomly generated by the source and conveyed via a shuttle to one of the two workstations (type A to the upper one and type B to the lower one) since each workstation can only produce one product type. In the simulation model, product part type A is coded with 1 and product part type B with 2 .

The quality control of the products is performed in a separate section, as can be seen in Figure 2. Those products that have some defect must return to one of the two workstations, depending on the type of product, via a shuttle based on the disposition of batch lots.
The faulty product parts are marked either with 10 (for type A) or with 20 (for type B). Those that have no defects leave the model at the sink. At the workstations, a set-up is necessary between the parts coming from the source and the faulty parts. As a consequence, there are high set-up times in the model [5].

The model used in our example is improved by comparison to the basic model of the tutorial (the parameter settings are shown in Figure 2). Therefore, the velocity (v) of the shuttle, as well as the buffer sizes, are improved, and a bulk conveyer organizes different batch sizes.

The simulation model is used to check the effects of different right-of-way strategies of the shuttle ("FIFO": First In First Out, or "Prio Corr.": prioritize correction route), as well as system loads at the source, and dif- 
ferent batch sizes on the possibility to reach a desired throughput per hour. In addition, the simulation model is used to examine the utilization rate of the two workstations and to uncover possible bottle-necks in the system.

\subsection{Response surface model-based LCCs}

Based on the described DOSIMIS-3 simulation model, we apply the procedure model from Section 2 to derive LCCs for the KPIs average throughput and utilization rate at the upper workstation. The KPI average throughput is the average number of parts processed per hour. The KPI utilization rate is measured as the time the workstation is in use in percentage, including setup times. Each of our simulation runs covers five days of simulation time with a four hour lead time. As an advantage of our metamodel-based approach, we can explore the behavior of these KPIs depending on various input variables, here the right-of-way strategy, the system load, and the batch size.

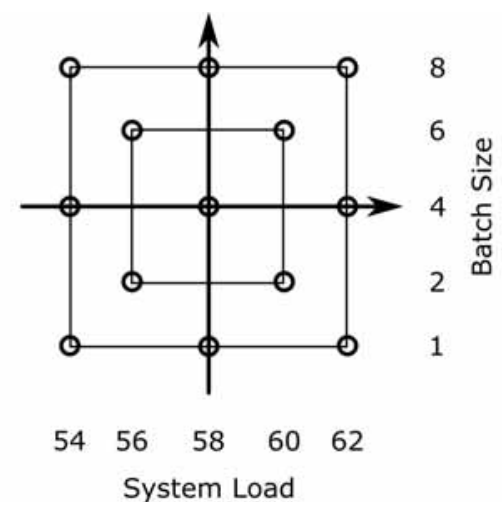

Figure 3: Settings of system load and batch size used in the statistical experimental design.

Table 1 contains the possible values these input variables can be set on in the simulation model. As the system load may be set on any value in the considered range from 54 to 62 , we get an infinite number of possible settings. Now, consider reducing the choice to every integer between 54 and 62, i.e., nine system loads. Together with the eight possible batch sizes in $\{1, \ldots, 8\}$ and the two considered right-of-way strategies, this leads to 144 possible combinations. As we plan to replicate each considered parameter setting three times with different random seeds, this would result in 432 simulation runs.

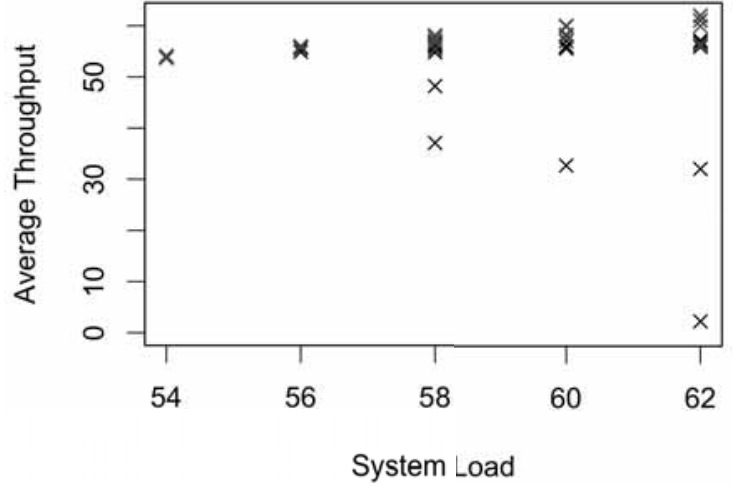

Figure 4: Scatterplot of average throughput vs. system load. The black points belong to the right-of-way strategy "FIFO" while the blue points belong to "Prio Corr.".

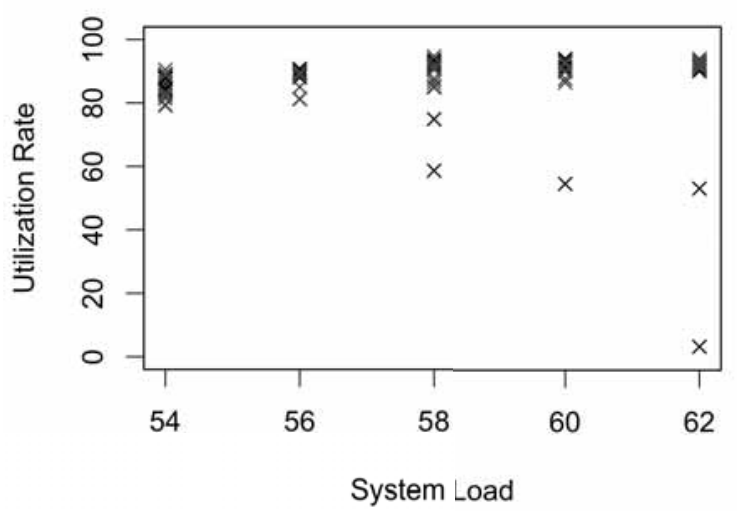

Figure 5: Scatterplot of utilization rates vs. system loads. The black points belong to right-of-way strategy "FIFO", the blue points to "Prio Corr.".

Instead, using the statistical Design of Experiments (DoE), we decide on a combination of a Central Composite Design (CCD) with axial points on the box and a full factorial design for two intermediate levels, which is to be conducted for each of the two right-of-way strategies. CCDs are classical design for the fitting of second-order response surface models [14]. A CCD consists of a full-factorial design of all combinations of high and low values (54 and 62 for system load and 1 and 8 for batch size), a center point (system load $=58$ and batch size $=4$ in our case) and so called axial or star points. In our chosen design, the axial points are on the box of the full-factorial design to cover the boundary area well. 

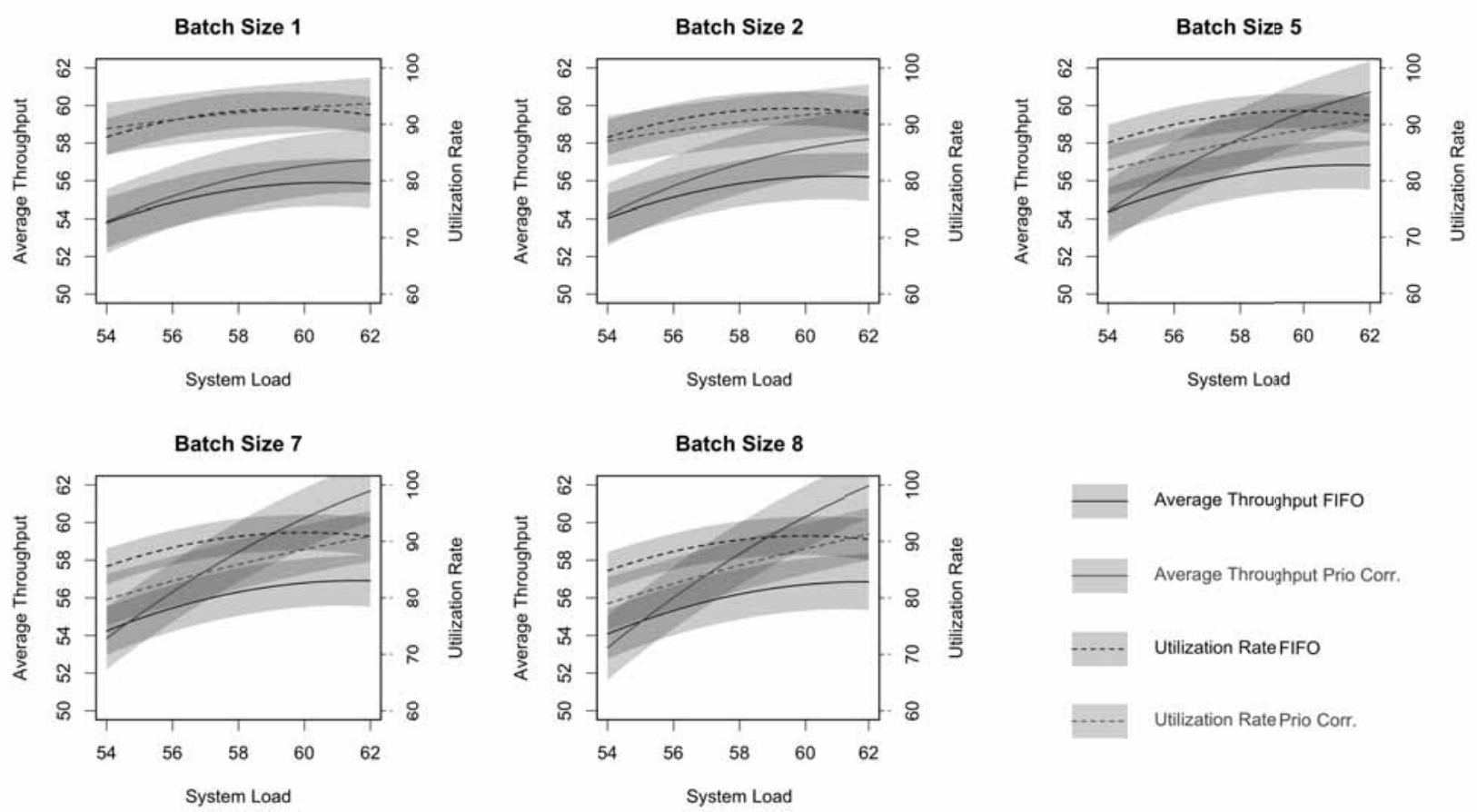

Figure 6: LCCs of average throughput and utilization rate of the upper workstation in dependence of system load, selected batch sizes, and both right-of-way strategies.

We extend the CCD by adding a full-factorial design on two medium levels (system load $=56 / 60$ and batch size $=2 / 6$ ) to achieve a more space-filling effect. Figure 3 indicates the 13 combinations of values for the system load and the batch size. These parameter settings are used for both right-of-way strategies, resulting in 26 different parameter settings in our experimental design. Each of these settings is replicated three times. Using a new random seed for each run, altogether 78 simulation runs are done, and the KPIs are observed.

Figure 4 and 5 show the average throughputs, and the utilization rates of the top workstation, respectively plotted against the system load. It is evident that most of the points' average throughputs are close to the related system loads. However, there are some deviating values with less than 50 parts per hour in average. For the utilization rate, a similar phenomenon can be observed. The outliers of both KPIs are identical and belong exclusively to the right-of-way strategy "FIFO". In these runs, a deadlock occurred. We omit the five runs in our building of LCCs by response surface models as they obviously display a very different pattern from the behavior of the remaining simulation results.
We build linear regression models for the KPIs by least-square estimation and separately for each rightof-way strategy. For the remaining input variables system load and batch size, a full second-order model of main effects, quadratic terms, and the interaction term is fitted [15]. The adjusted determination of coefficients $\left(R^{2}\right)$ measures the goodness-of-fit of a regression model with possible values between 0 and 1 . For the four fitted models, $R^{2}$ takes values between 0.84 and 0.95 , indicating a good fit in each case.

Figure 6 contains LCCs for both average throughput and utilization rate of the upper workstation. They consist of the models' predictions (the lines) together with the $99 \%$ prediction intervals (the ribbons). Note, that we can generate these predictions for all parameter settings within the domain. We are especially not restricted to batch sizes 1, 2, 4, 6, and 8 only. Here, we display LCCs for small batch sizes (1 and 2), for a medium batch size (5) and for batch sizes at the upper end of our considered range (7 and 8). 
For example, the solid line depicted in Figure 6 for the average throughput in case of "Prio Corr." as rightof-way strategy is calculated by the estimated prediction equation

$$
\begin{aligned}
& \text { average throughput } \text { Prio Corr. }=-105.766 \\
& +5.345 \cdot \text { system load }-4.612 \cdot \text { batch size } \\
& -0.043 \cdot \text { system load }{ }^{2}-0.070 \cdot \text { batch } \text { size }^{2} \\
& +0.096 \cdot \text { system load } \cdot \text { batch size. }
\end{aligned}
$$

It is obvious that the LCCs vary between batch sizes and right-of-way strategies. They show what can be achieved with the different considered variations of the simulated production process. For example, in case that a system load of 54 parts per hour has to be processed, the average throughput should also equal 54. This can be achieved with both right-of-way strategies using only a batch size of 1 . However, for larger system loads, "FIFO" as a right-of-way strategy is not successful. With higher required average throughputs, "Prio Corr." needs to be chosen along with increasing batch sizes. E.g., a system load of 58 requires at least batch size 5. With an even higher batch size, the utilization rate can be reduced. The upper limit of considered system loads can only successfully be processed by using at least batch size 8 .

\subsection{Validation of the procedure model}

To validate our procedure model in the case of the DOSIMIS-3 simulation model, we conduct further simulation runs. We extend our data set to contain each combination of system loads with an integer value between 54 and 62, batch sizes 1, 2, 4, 6, and 8, as well as the two right-of-way strategies, replicated three times. Altogether, we end up with 270 simulation runs.

In Figure 7, we have plotted the data points against the model's predictions for the average throughput. Within the additional simulation runs, seven more deadlocks for right-of-way strategy "FIFO" occurred, which we exclude for clarity's sake.

We can see that only a few points do not lie within the prediction intervals, e.g., for "FIFO", a system load of 61 and batch sizes of 4 or 6 . The three replications for each of these two locations, exhibit a greater variance than most of the other replications, which cannot be covered by the model. Altogether, the model shows a good prediction accuracy.
Figure 8 shows the predictions of the utilization rate of the upper workstation for the same system loads along with the $99 \%$ prediction intervals. For the rightof-way strategy "Prio Corr.", all of the points lie within the prediction interval. For "FIFO" again, a few points lie outside the interval. However, the overall behavior of the model seems more than plausible.

\section{Summary and outlook}

In this paper, we have presented a new procedure model to obtain LCCs based on simulation models and statistical metamodeling. Although only a few simulation runs are required, it is possible to describe the system behavior and the resulting KPIs depending on different system configurations. Using statistical models also allows for quantifying the system-inherent uncertainty. With a first example of application, the basic feasibility and usefulness of the procedure model are shown. Based on these results, other research is planned to prove the applicability and the general validity of the procedure model, also for more complex systems and further KPIs. Extensions of the approach and new methodological developments also appear to be useful with regard to statistical modeling. For example, the prediction intervals capturing the uncertainty are symmetrical in the presented simulation model, although, the average throughput, in particular, cannot, in general, be higher than the system load. Also, the occurring deadlocks are not pursued further in our investigations. It is conceivable to define the probability of the occurrence of deadlocks as a KPI through suitable metamodels and to represent it in LCCs.

\section{References}

[1] Nyhuis P, Wiendahl, H-P. Fundamentals of Production Logistics. Springer: Heidelberg; 2009.

[2] Hopp WJ, Spearman ML. Factory physics. Foundations of manufacturing management. Irwin/McGraw-Hill: Boston; 2001.

[3] Gutenschwager K, Rabe M, Spieckermann S, Wenzel S. Simulation in Produktion und Logistik. Grundlagen und Anwendungen. Berlin: Springer Vieweg; 2017.

[4] Bracht U, Geckler D, Wenzel S. Digitale Fabrik. Methoden und Praxisbeispiele. Berlin: Springer Vieweg; 2018.

[5] SDZ GmbH Tutorial. Part 1 \& 2. Version 8.1. 2020. Online https://www.sdz.de/downloads/, 28.02.2020. 

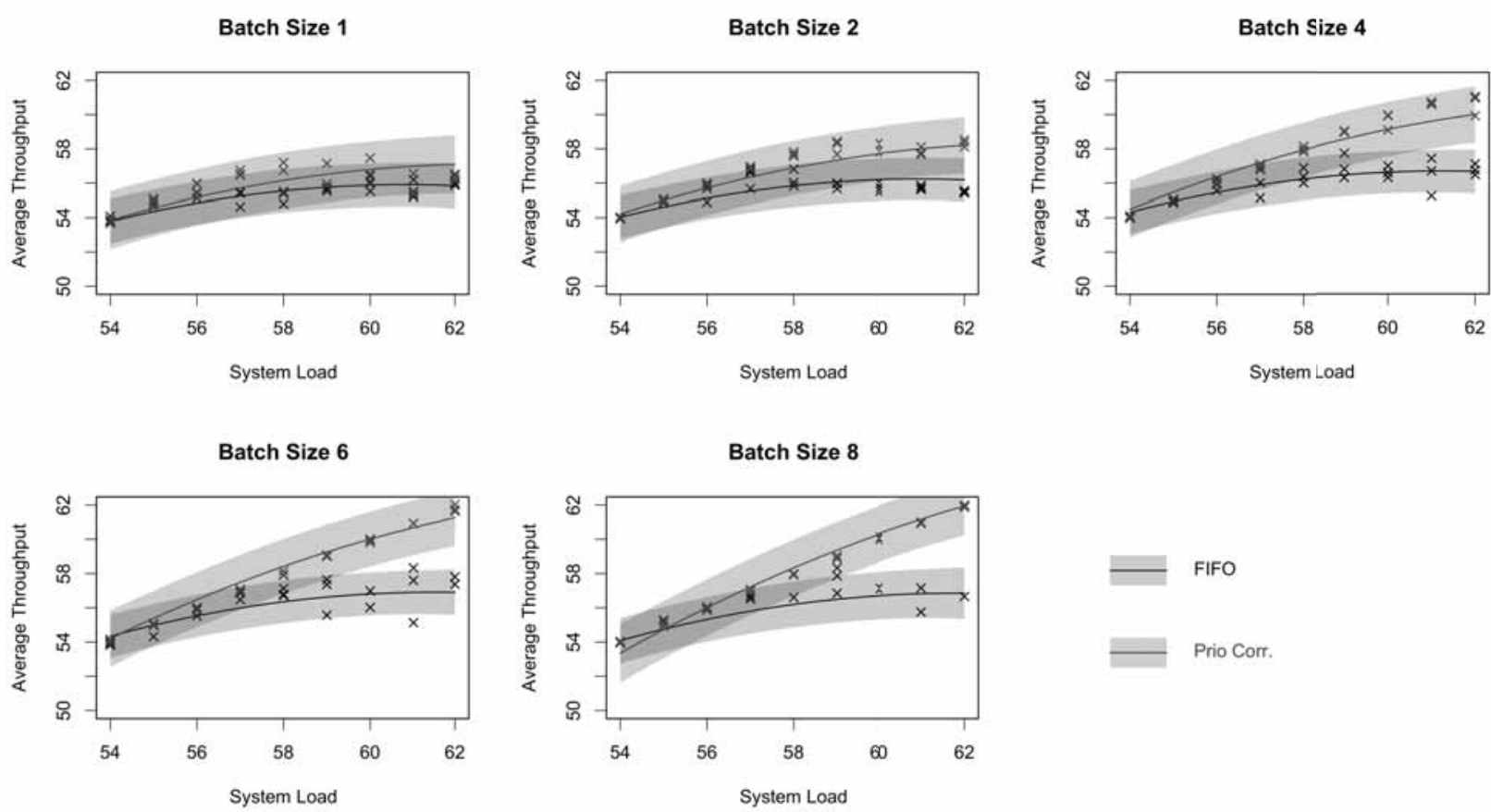

Figure 7: LCCs for average throughput with $99 \%$ prediction interval and all simulated data points.
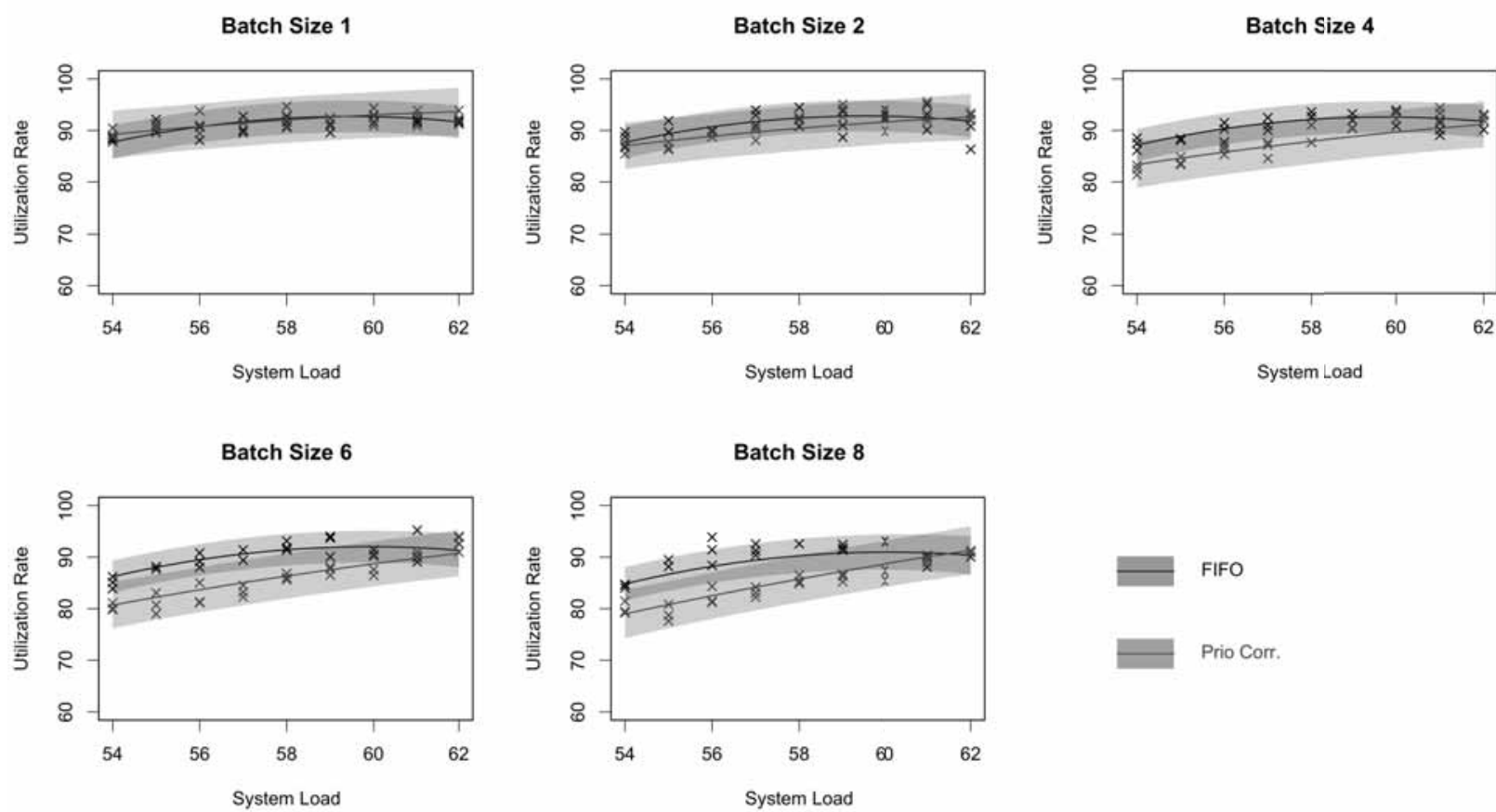

Figure 8: LCCs for utilization rate with $99 \%$ prediction interval and all simulated data points. 
[6] Nyhuis P, Wiendahl H-P. Logistische Kennlinien. Grundlagen, Werkzeuge und Anwendungen. Berlin: Springer; 2003.

[7] Nyhuis P, Wiendahl H-P. Logistic Production Operating Curves - Basic Model of the Theory of Logistic Operating Curves. CIRP Annals. 2006; 55(1): 441-444.

[8] Nyhuis, P. Practical Applications of Logistic Operating Curves. CIRP Annals. 2007; 55(1): 483-486.

[9] Weigert, G. Operating Curves of Manufacturing Systems: A Theoretical Discourse. In: Azevedo, A, editor. Advances in Sustainable and Competitive Manufacturing Systems. Lecture Notes in Mechanical Engineering. Heidelberg: Springer; 2013. 887-897.

[10] VDI 3633 Blatt 1:2014-12. Simulation von Logistik-, Materialfluss und Produktionssystemen; Grundlagen (Simulation of systems in materials handling; Logistics and production). Berlin: Beuth Verlag. (A list of the currently available parts in this series of standards is available on the Internet at www.vdi.de/3633.)
[11] Wenzel S, Weiß M, Collisi-Böhmer S, Pitsch H, Rose O. Qualitätskriterien für die Simulation in Produktion und Logistik. Berlin Heidelberg: Springer; 2008.

[12] Wenzel, S. Simulation logistischer Systeme. In: Tempelmeier, H, editor. Modellierung logistischer Systeme. Berlin: Springer-Vieweg; 2018. 1-34.

[13] Santner TJ, Williams BJ, Notz WI. The Design and Analysis of Computer Experiments. 2nd ed. New York: Springer; 2018.

[14] Myers RH, Montgomery DC, Anderson-Cook CM. Response Surface Methodology: Process and Product Optimization Using Designed Experiments. 4th ed. Hoboken, New Jersey: Wiley; 2016.

[15] Fahrmeir L, Kneib T, Lang S, Marx B. Regression: Models, Methods and Applications. Berlin Heidelberg; Springer; 2013. 